\title{
Galactose Modified Liposomes for Effective Co-Delivery of Doxorubicin and Combretastatin A4
}

This article was published in the following Dove Press journal: International Journal of Nanomedicine

\author{
Bo Lian $1, *$ \\ Hua Wei ${ }^{2} *$ \\ Ruiyan $\operatorname{Pan}^{3, *}$ \\ Jingui Sun ${ }^{4}$ \\ Bo Zhang ${ }^{3}$ \\ Jingliang $\mathrm{Wu}\left(\mathbb{D}^{1}\right.$ \\ Xiujie $\mathrm{Li}^{1}$ \\ Guixiang Tian'
}

'School of Bioscience and Technology, Weifang Medical University, Weifang 261053, People's Republic of China; ${ }^{2}$ Department of Endocrinology, ShouGuang Peoples' Hospital, Weifang 262700, People's Republic of China; ${ }^{3}$ School of Pharmacy, Weifang Medical University, Weifang 26I053, People's Republic of China; ${ }^{4}$ Department of Oncology, ShouGuang Peoples' Hospital, Weifang 262700, People's Republic of China

*These authors contributed equally to this work
Correspondence: Xiujie Li Email lixiujie@wfmc.edu.cn

Guixiang Tian

School of Bioscience and Technology,

Weifang Medical University, Weifang

261053, People's Republic of China

Email gxtian2008@163.com
Background: Tumor angiogenesis plays a crucial role in tumor development, and recent efforts have been focused on combining proapoptotic and antiangiogenic activities to enhance antitumor therapy.

Methods: In this study, galactose-modified liposomes (Gal-LPs) were prepared for codelivery of doxorubicin (DOX) and combretastatin A4 phosphate (CA4P). The co-cultured system composed of BEL-7402 and human umbilical vein endothelial cells (HUVEC) cells was established to effectively evaluate in vitro anti-tumor activity through cell viability and cell migration assay. Furthermore, both in vivo bio-distribution and anti-hepatoma effect of DOX\&CA4P/Gal-LPs were investigated on H22 tumor cell-bearing mice.

Results: The results showed that DOX\&CA4P/Gal-LPs were spherical with a mean particle size of $143 \mathrm{~nm}$, and could readily be taken up by BEL-7402 cells. Compared with a mixture of free DOX and CA4P, the DOX\&CA4P/Gal-LPs were more effective in inhibiting cell migration and exhibited stronger cytotoxicity against BEL-7402 cells alone or a co-cultured system. The in vitro studies showed that the co-cultured system was a more effective model to evaluate the anti-tumor activity of combination therapy. Moreover, DOX\&CA4P/Gal-LPs exhibited a greater anti-hepatoma effect than other drug formulations, indicating that Gal-LPs could promote drug accumulation in the tumor region and improve the anti-tumor activity.

Conclusion: Gal-LPs co-loaded with chemotherapeutic and antiangiogenic drugs are a promising strategy for anti-hepatoma therapy.

Keywords: liposomes, anti-hepatoma, co-delivery, combination therapy

\section{Introduction}

More and more evidence has been provided that traditional therapy based on a single anti-tumor drug fails to eliminate the tumor due to the complexity of tumor development. ${ }^{1}$ Accordingly, combination therapy has been widely applied as an effective strategy by combining two or more drugs via different antitumor mechanisms. ${ }^{2,3}$ Nowadays, it has attracted great attention in the combination of chemotherapeutic and anti-angiogenic agents for an enhanced anti-tumor effect. ${ }^{4,5}$ In this strategy, the antiangiogenic drugs could induce the blood vessel shutdown by targeting the abnormal vasculature, while the chemotherapeutic drugs could inhibit tumor development by pro-apoptotic activities, herein leading to a superior antitumor effect. ${ }^{6-8}$

The tumor angiogenesis plays an important role in the process of tumor development and metastasis. Anti-angiogenesis therapy has emerged as a potential strategy in the inhibition of tumor growth by blocking the nutrient supply. ${ }^{9,10}$ Recent researchhas been 
focused on the development of new anti-angiogenic agents. $^{11,12}$ Among these, combretastatin-A4 (CA4), a compound from combretum caffrum, is a promising antiangiogenic drug, which could induce vasoconstriction and collapse of blood vessels by targeting the abnormal vascular system. ${ }^{13-15}$ Therefore, combining CA4 and chemotherapeutic drug, such as doxorubicin (DOX), is expected to inhibit tumor development by pro-apoptotic and anti-angiogenic activities simultaneously.

However, traditional combinational therapy by a mixture of free drugs has exhibited many disadvantages in clinics, such as systemic toxicity, lack of selectivity, and low bioavailability. ${ }^{16,17}$ In order to improve the selectivity and reduce the systemic side-effects, it is necessary to prepare an efficient co-loaded drug delivery system, which could carry two or more drugs into the tumor region, and effectively be taken up by tumor cells. ${ }^{13,18}$ In the past few decades, liposomes (LPs) modified by special moieties, such as antibodies, sugars, and various ligands, have been utilized for targeting delivery of anti-tumor drugs. ${ }^{19-21}$ Compared with normal liver tissue, the asialoglycoprotein-receptor (ASGP-R) is over-expressed on the surface of tumor cells in Hepatocellular Carcinoma (HCC). ${ }^{22,23}$ The liposomes modified by galactose (Gal), a molecular specially binding to ASGP-R, were expected to improve the efficacy of combination therapy.

In this study, Gal-modified liposomes were prepared for co-delivery of DOX and CA4P to inhibit HCC development through pro-apoptotic and anti-angiogenic activities (Figure 1). A co-cultured system composed of BEL-7402 and HUVEC cells was established to investigate the combination therapeutic effects in vitro. The in vivo real-time near-infrared fluorescence (NIRF) imaging was performed to explore the targeting properties of Gal-LPs, and H22 tumor-bearing mice were used to evaluate the anti-hepatoma efficacy of DOX\&CA4P co-loaded liposomes in vivo.

\section{Materials and Methods}

\section{Materials}

CA4P (MW: 440.30), Lactobionic acid (LA, MW: 358.3) and cholesterol were purchased from Beijing Innochem Co., Ltd. (China). L- $\alpha$-phosphatidylcholine, Thiazolyl blue tetrazolium bromide (MTT, MW:414.3), fetal bovine serum (FBS) and 4',6-Diamidino-2-phenylindole (DAPI, MW:350.2) were obtained from Beijing Solarbio Co., Ltd. (China). DOX was obtained from Shanxi Powerdone Pharmaceutical Co., Ltd (China). DSPE-PEG-NHS was
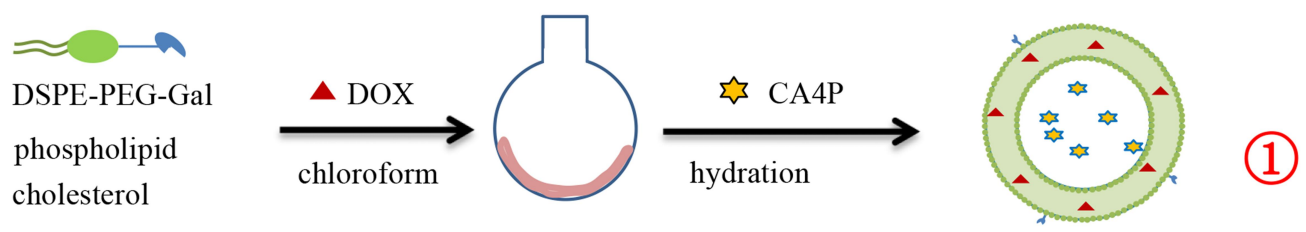

lipid thin-film

DOX\&CA4P/Gal-LPs

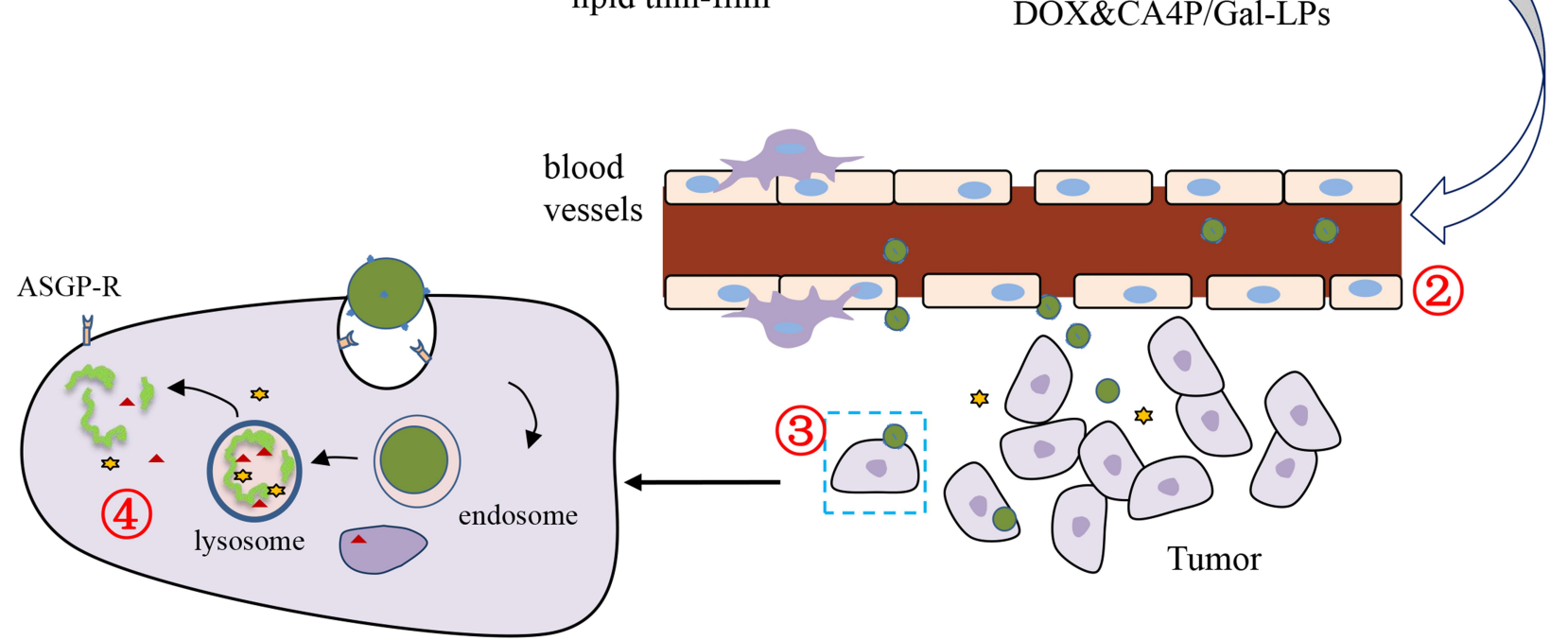

Figure I Schematic illustration of combination therapy. (1)Preparation of DOX\&CA4P/Gal-LPs, (2)Liver-targeting delivery via blood cycle, (3)Cellular uptake mediated by ASGP-R, and (4)IDrug release in tumor cells. 
obtained from Xi'an Ruixi Technology Co., Ltd. (China). All other chemicals were of analytical grade.

\section{Cell Culture}

Human hepatic cell line (BEL-7402), Hela, and HUVEC cells were obtained from Beijing Bnbio Research Institute (China). An in vitro co-cultured system was established by mixing BEL7402 and HUVEC cells in RPMI 1640 medium at a ratio of 5:1.

\section{Animals}

Female BALB/c mice (age: 6 8 weeks; weight: approximately 18-20 g) were purchased from Jinan Pengyue Experimental Animal Breeding (China); All animal experiments were approved by the WFMU Animal Research Ethics Committee, approval No. 2019-045. Care and handling of the animals were in strict compliance with the "Guide for the Care and Use of Laboratory Animals" of China.

\section{Preparation and Characteristics of Co-Loaded Liposomes}

DSPE-PEG-Gal was synthesized by a two-step reaction between DSPE-PEG-NHS and LA according to previous studies. ${ }^{24}$ Gal-LPs were prepared by thin-film evaporation. Briefly, L- $\alpha$-phosphatidylcholine, cholesterol, and DSPEPEG-Gal were mixed in chloroform at a mass ratio of 12:4:1. The particle size, $\zeta$ potential, and polydispersing index (PdI) of Gal-LPs were characterized using dynamic light scattering method with a wavelength of $633 \mathrm{~nm}$ at $25^{\circ} \mathrm{C}$. The morphology of the blank LPs and Gal-LPs was observed by transmission electron microscopy (TEM, HT7700, HITACHI, Japan). Briefly, one drop of Gal-LPs solution was placed on a copper grid. After drying, the sample was stained with $2 \%$ phosphotungstic acid, and then observed under TEM.

DOX\&CA4P/Gal-LPs were prepared for combination therapy. In brief, a mixed solution of L- $\alpha$ phosphatidylcholine, cholesterol, and DOX in chloroform was evaporated to form a dry lipid film. The CA4P solution in PBS was added to hydrate the lipid film, followed by sonicating the mixture solution for smaller particle size. Meanwhile, DOX\&CA4P/LPs were prepared for control. The characteristics of drug-loaded LPs were tested as before. ${ }^{25}$ To evaluate the stability of DOX\&CA4P/Gal-LPs, the samples were dispersed in serum-free RPMI 1640 medium. The particle size and $\zeta$ potential were measured using dynamic light scattering (Zetasizer Nano ZS90, Malvern, England) for 15 days. CA4P was measured using highperformance liquid chromatography (HPLC, Agilent1260, USA). The mobile phase was composed of $60 \%$ of methanol and $40 \%$ of $0.05 \mathrm{M} \mathrm{KH}_{2} \mathrm{PO} 4$ solution, and flow rate was fixed at $1 \mathrm{~mL} / \mathrm{min}$. In total, $20 \mu \mathrm{L}$ of sample was injected and the total run time was 8 minutes. DOX was detected at 490 $\mathrm{nm}$ using a UV/VIS spectrophotometer (UV-8000, Shanghai, China). The encapsulation efficiency (EE) and loading efficacy (DL) were measured using high-performance liquid chromatography (HPLC), and calculated according to the following formulae: $\mathrm{EE}=\mathrm{Ws} / \mathrm{Wa} \times 100 \%$, where Ws stands for the amounts of DOX or CA4P loaded in the LPs, while Wa means the initial added amounts of DOX or CA4P added. These studies were repeated in triplicate.

\section{In vitro Drug Release from LPs}

The release profiles of DOX and CA4P from LPs were investigated by dialysis method. DOX\&CA4P/Gal-LPs were dispersed in PBS ( $\mathrm{pH}$ 7.4), and the solution was transferred into a dialysis bag (MWCO of $3.5 \mathrm{kDa}$ ). Then, the dialysis bag was placed in a bottle containing $20 \mathrm{~mL}$ of release medium (PBS buffer solution containing 0.5\% Tween 80 ). The release system is kept in a shaking incubator under a shaking speed of $100 \mathrm{rpm}$ at $37^{\circ} \mathrm{C}$. At predetermined time intervals $(0.5,1,2,4,8,12,24$, and 48 hours), $2 \mathrm{~mL}$ of medium was taken out and the same amounts of free release solution were added. The concentrations of DOX and CA4P were measured based on their standard curve, respectively, and their cumulative release percentages (Er) were calculated by the following equation:

$$
\operatorname{Er}(\%)=\frac{\mathrm{V}_{\mathrm{r}} \sum_{1}^{\mathrm{n}-1} \mathrm{C}_{\mathrm{i}}+\mathrm{V}_{\mathrm{o}} \mathrm{C}_{\mathrm{n}}}{\mathrm{M}_{\mathrm{d}}} \times 100 \%
$$

Where $\mathrm{M}_{\mathrm{d}}$ is the amount of DOX or CA4P in the LPs, $\mathrm{V}_{0}$ stands for the whole volume of release medium, $\mathrm{C}_{\mathrm{i}}$ is the concentration of drugs in release medium, and $V_{r}$ stands for the volume of the replaced medium.

\section{In vitro Cellular Uptake}

In vitro cellular uptake was performed to evaluate the active-targeting properties of Gal-LPs. ${ }^{26}$ In brief, BEL7402 or Hela cells were seeded in 6-well plates at a density of $5 \times 10^{4}$ cells $/ \mathrm{mL}$ at $37^{\circ} \mathrm{C}$. After $18-24$ hours of incubation, the media were replaced with $2 \mathrm{~mL}$ of fresh RPMI-1640 medium containing DOX\&CA4P/LPs or DOX\&CA4P/Gal-LPs (at the equivalent DOX concentration of $5 \mu \mathrm{g} / \mathrm{mL}$ ) for 4 hours of incubation, respectively. 
Afterwards, the media were discarded, and the cells were washed with cold PBS. Furthermore, the cells were dyed by DAPI for 10 minutes and washed by PBS. Finally, the cellular uptake of drug-loaded LPs was visualized under a confocal microscope (TCS SP8, Leica, Germany) with the excitation wavelength at $361 \mathrm{~nm}$ for DAPI and $485 \mathrm{~nm}$ for DOX.

\section{In vitro Cytotoxicity Analysis}

To evaluate the cytotoxicity of drug-loaded LPs, the MTT assay was performed against BEL-7402 cells alone or BEL7402/HUVEC co-cultured system, respectively. BEL7402 cells or co-cultured cells (the ratio of BEL7402 to HUVEC at 5:1) were seeded in 96-well plates at a density of 5,000 cells/well for $18-24$ hours. Then the media were replaced with serum-free fresh media containing DOX, a mixture of DOX and CA4P, DOX\&CA4P/LPs, and DOX\&CA4P/Gal-LPs, respectively. The DOX concentrations were set to be $0.01,0.1,1,2$, and $5 \mu \mathrm{g} / \mathrm{mL}$. After 48 hours of incubation, $10 \mu \mathrm{L}$ of MTT solution $(0.5 \mathrm{mg} / \mathrm{mL})$ was added to each well, and the cells were incubated for another 4 hours. Then, the media were discarded, and 150 $\mu \mathrm{L}$ of DMSO was added. The fluorescence intensity at 570 $\mathrm{nm}$ was recorded using a micro plate reader (Bio-Rad 500, USA).

\section{In vitro Cell Migration Assays}

A 2D wound healing assay was performed to evaluate the effects of different drug formulations on cellular mobility in the Bel-7402 cells alone or BEL7402/HUVEC co-cultured system, respectively. In order to distinguish different cells in a co-cultured system, the 5.6-carboxyfluorescein diacetate, succinimidyl ester (CFSE)-labeled HUVEC cells model was established. ${ }^{27}$ BEL-7402 cells or co-cultured system were seeded in a 6-well plate at a density of $5 \times 10^{4}$ cells per well. After forming a confluent monolayer, the cells were scraped by a p200 pipet tip to create an empty gap. The media were replaced by fresh media with different drug formulations. The cells were imaged at 0 and 24 hours using microscopy, and the degree of wound healing was calculated by the following equation:

wound healing rate $=$ (wound area on 0 hour - wound area on $\mathrm{n}$ hours $) /($ wound area on 0 hour $) \times 100 \%$, where $\mathrm{n}=24$ hours post-wounding.

\section{In vivo Biodistribution}

To investigate the liver-targeting property of Gal-LPs, $\mathrm{DiR} / \mathrm{Gal}-\mathrm{LPs}$ were prepared to track the in vivo biodistribution of drugs. Briefly, the H22 tumor-bearing mice were established through injecting $\mathrm{H} 22$ cells subcutaneously on the right flank of female BALB/c mice. When the tumor volume reached $200 \mathrm{~mm}^{3}$, these mice were randomly divided into two groups and injected intravenously via the tail vein with free DiR or DiR/Gal-LPs. The in vivo distribution of DiR was observed by IVIS ${ }^{\circledR}$ Kinetics Optical System (PerkinElmer, USA). The excitation and emission wavelength were set at $710 \mathrm{~nm}$ and 790 $\mathrm{nm}$, respectively.

\section{In vivo Antitumor Efficacy}

$\mathrm{H} 22$ cell-bearing BALB/c mice were established to investigate the anti-hepatoma efficacy of different drug formulations. In brief, $0.2 \mathrm{~mL}$ of $\mathrm{H} 22$ cells at a concentration of $4 \times 10^{6}$ cells $/ \mathrm{mL}$ was subcutaneously inoculated into the flanks of BALB/c mice. When the tumor volume reached $100 \mathrm{~mm}^{3}$, the mice were randomized into five groups: saline (control), free DOX, mixture of DOX and CA4P, DOX\&CA4P/LPs, and DOX\&CA4P/Gal-LPs. The mice were injected through the tail vein every 2 days, and the dosage of DOX was set at $5 \mathrm{mg} / \mathrm{kg}$. The mice were weighed, and tumor size was measured every 2 days. The tumor volumes were calculated according to the following equation:

$$
V=a \times b^{2} / 2
$$

where $\mathrm{a}$ and $\mathrm{b}$ represent the long axis and short axis, respectively. After 2 weeks of administration, mice were euthanized, and the tumors were harvested and weighed. Finally, the growth-rate of inhibition (GRI) was calculated by the following equation:

$$
\mathrm{GRI}=\left(1-\mathrm{W}_{\mathrm{S}} \backslash \mathrm{W}_{\mathrm{C}}\right) \times 100 \%
$$

where Ws is the tumor weight of the drug-treated group, and $\mathrm{Wc}$ is the tumor weight of the control group.

\section{Immunohistochemistry and Histological Staining}

At the end of drug administration, the major organs were harvested for immunohistochemistry and histological staining. In brief, the tumors and organs were fixed with $4 \%$ PBS buffered paraformaldehyde, and embedded in paraffin. The paraffin-embedded samples were cut into slices at $4 \mu \mathrm{m}$ thickness using an ultrathin slicer, and histopathological changes were investigated by hematoxylin and eosin (H\&E) staining via an inverted microscope (IX51; Olympus, Tokyo, Japan). To evaluate the anti-angiogenic 
effect of combination therapy, the microvascular density of CD31 was measured by immunohistochemistry assay.

\section{Statistical Analysis}

All experimental data in this study were expressed as mean \pm standard deviation ( $\mathrm{SD}, \mathrm{n}=3$ ). Statistical significances were determined through a Student's $t$-test, and $P<0.05$ was considered as statistically significant.

\section{Results and Discussion}

\section{Characterizations of Drug-Loaded Liposomes}

The results showed that DOX and CA4P could successfully encapsulate into Gal-LPs. The EE\% values of DOX and CA4P were $86.74 \%$ and $52.43 \%$, respectively. The DL\% were $5.62 \%$ and $2.03 \%$, respectively. The mean particle size and zeta-potential of DOX\&CA4P/Gal-LPs were 143.01 $\pm 7.32 \mathrm{~nm}$ and $-24.18 \pm 3.45 \mathrm{mV}$, respectively. And those of DOX\&CA4P/LPs were $137.12 \pm 4.59 \mathrm{~nm}$ and $-25.67 \pm 2.91$ $\mathrm{mV}$, respectively. TEM images indicated that the shape of drug-loaded LPs was spherical (Figure 2A and B). Moreover, the stability of DOX\&CA4P/Gal-LPs was determined in RPMI-1640 medium. There was no significant difference in the mean particle size and zeta-potential within 2 weeks, indicating that the liposomal formulation was stable under physiological conditions (Figure 2C). ${ }^{28}$

In vitro drug release from Gal-LPs was evaluated in the media at $37^{\circ} \mathrm{C}$. Figure $2 \mathrm{D}$ shows that DOX and CA4P exhibited time-dependent release within 48 hours. Approximately $30 \%$ of CA4P and $20 \%$ of DOX was released from Gal-LPs at 4 hours, which was similar in the LPs. No obvious burst release was observed, indicating that nano-sized liposomes have sustained release properties. $^{29}$ At 48 hours, the cumulative drug releases from Gal-LPs were about $75 \%$ of CA4P and $45 \%$ of DOX, suggesting that, compared with hydrophobic DOX, CA4P has a higher release rate due to its hydrophobicity.

\section{In vitro Cellular Uptake}

To analyze whether Gal-LPs is capable of active-targeting delivery of DOX into tumor cells, the cellular uptake of DOX was investigated in both BEL-7402 and Hela cells, respectively. The auto-fluorescence (Red) of DOX was used to track the distribution of DOX in cells, and the cell nuclei were dyed by DAPI (blue). As shown in Figure 3A and B, stronger fluorescence signals were observed in the cytoplasm and nuclei of BEl-7402 cells treated with DOX\&CA4P/GalLPs in comparison with the DOX\&CA4P/LPs, indicating that Gal-LPs could deliver more DOX into BEL-7402 cells than Gal-free liposomes. However, no obvious difference was observed in the Hela cells (Figure 3C-E). This was due to the different express level of ASGP-R between the BEL-7402 and Hela cells. ASGP-R was over-expressed on the cytomembrane of BEL-7402, therefore Gal-LPs could improve cellular uptake of DOX via ASGP-R-mediated endocytosis. ${ }^{30,31}$ The results suggested that introduction of
A

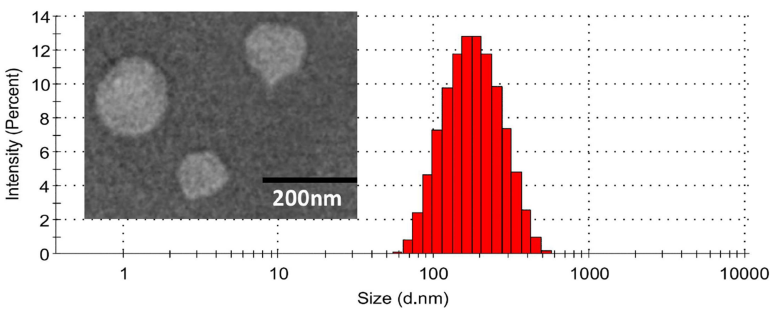

C

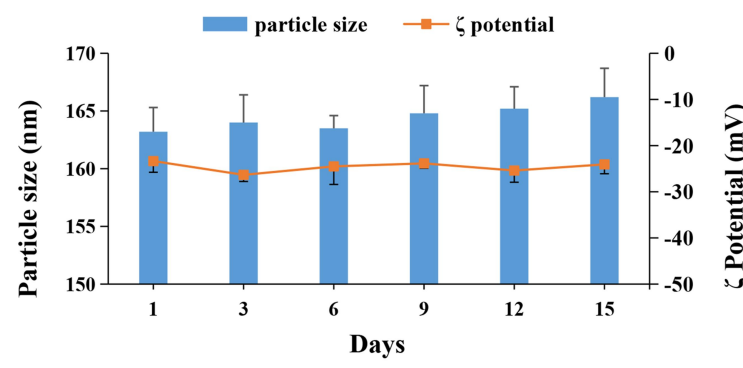

B

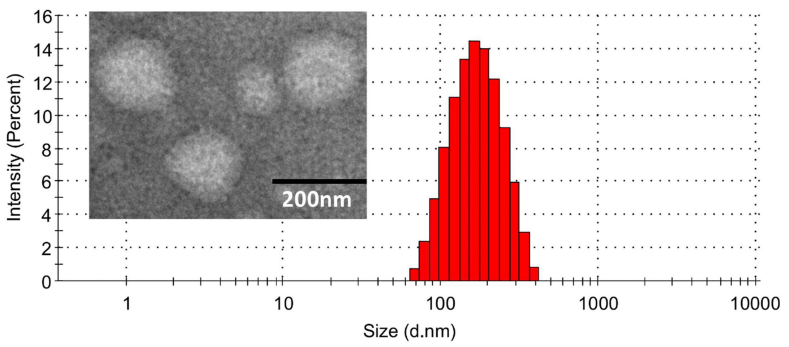

D

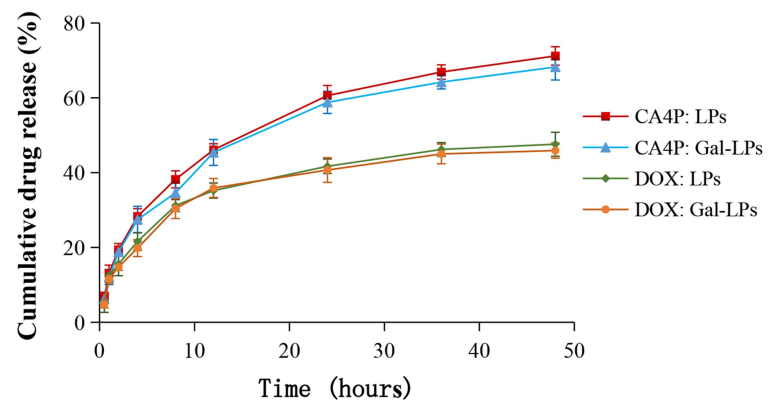

Figure 2 The characteristics of co-loaded liposomes. The particle-size distribution and TEM image of (A) DOX\&CA4P/LPs and (B) DOX\&CA4P/Gal-LPs. (C) The change of particle size and $\zeta$ potential for 15 days in RPIM 1640 medium. (D) In vitro drug release of DOX and CA4P from liposomes (LPs and Gal-LPs) through dialysis in PBS buffer at pH 7.4. 


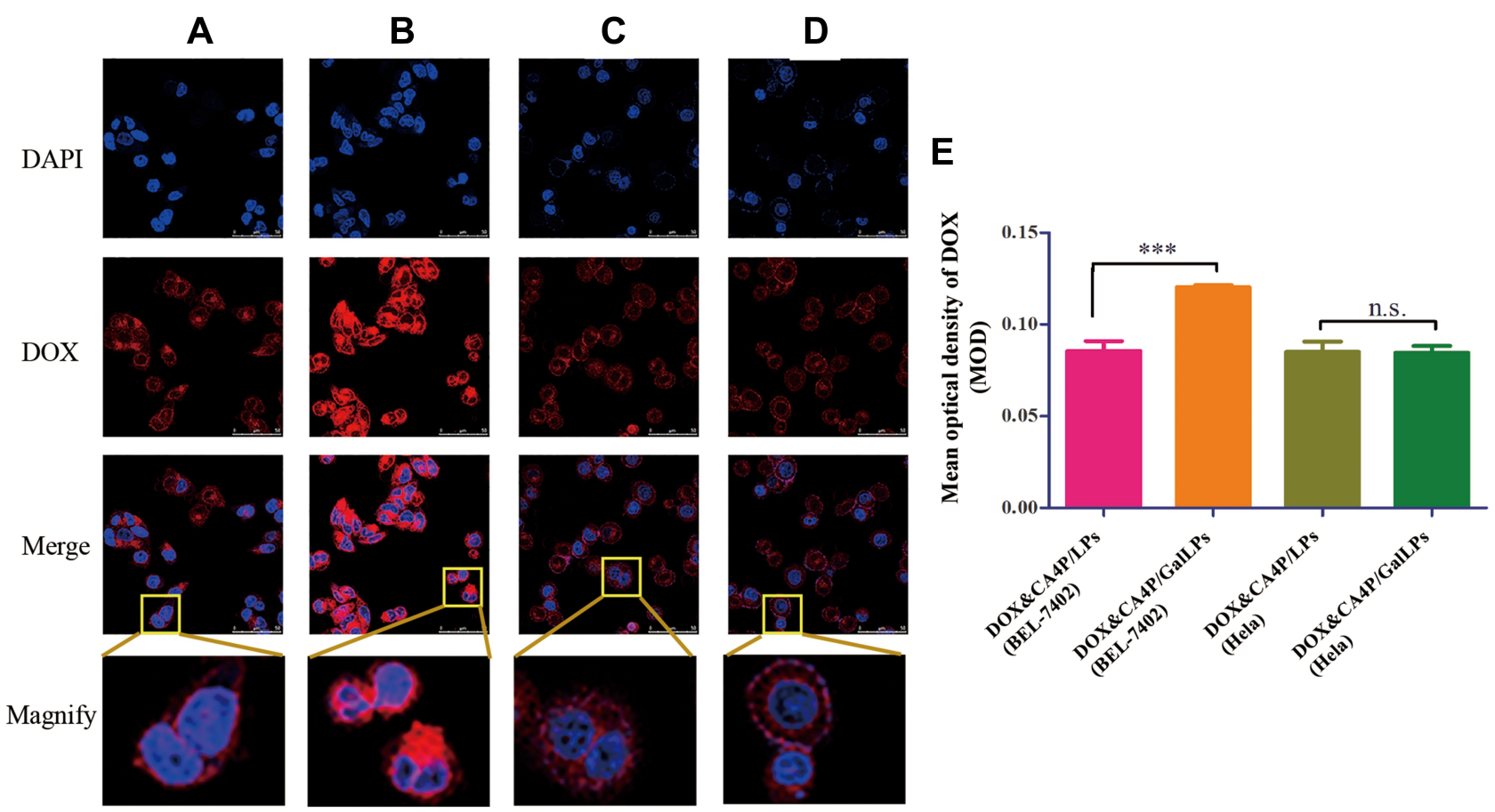

Figure 3 Confocal images of Bel-7402 cells incubated with DOX\&CA4P/LPs (A) and DOX\&CA4P/Gal-LPs (B); Hela cells incubated with DOX\&CA4P/LPs (C) and DOX\&CA4P/Gal-LPs (D). The quantitative data of fluorescence intensity in the cytoplasm (E). ***Value presents statistically significant difference $(P<0.00 \mathrm{I})$, and no statistically significant difference was presented using n.s.

Gal ligand on LPs could enhance the cell internalization of drug-loaded LPs, and Gal-LPs have the potential to carry anti-tumor drugs for anti-hepatoma therapy.

\section{Cytotoxicity Assay}

In this study, the combination therapy based on liposomes was designed by co-delivering two drugs with proapoptotic (DOX) and anti-angiogenic (CA4P) activities. However, It is difficult to exactly evaluate the anti-tumor effect by tumor cells alone in vitro due to different antitumor mechanisms of two drugs. ${ }^{32}$ In order to mimic the interaction between tumor cells and vascular endothelial cells in the tumor environment, we established a cocultured system which was composed of Bel-7402 and HUVEC cells to evaluate the anti-proliferation and antiangiogenesis effects of the combination treatment.

To evaluate the cytotoxicity of different drug formulations, cellular viability against BEL-7402 cells or BEL7402/HUVEC co-cultured system was investigated by MTT assay (Figure 4A). As shown in Figure 4B, the cellular viability of blank liposomes and Gal-LPs was over $85 \%$ at all concentrations $(10-500 \mathrm{mg} / \mathrm{mL})$, indicating that Gal-LPs had good bio-compatibility and were suitable to be used as the nanocarriers for anti-tumor drugs. ${ }^{32}$ After 48 hours of incubation, free DOX, mixture of DOX and CA4P,
DOX\&CA4/LPs, and DOX\&CA4/Gal-LPs showed dosedependent cytotoxicity against BEL7402 cells alone or a cocultured system. Compared with treatment of free DOX, the combined formulations against co-cultured systems showed higher cytotoxicity, indicating that the combination of chemotherapeutic and antiangiogenic drugs exhibited stronger cytotoxicity than a single drug (Figure 4C). Moreover, similar to the BEL-7402 cells alone, the cellular viability of DOX\&CA4/Gal-LPs was lower than that of DOX\&CA4/ LPs in the co-cultured system. The results suggested that introduction of Gal molecules on the surface of liposomes could increase the accumulation of DOX in cells, and thus lead to enhanced cytotoxicity, which was consistent with the analysis of cellular uptake. ${ }^{33,34}$

\section{In vitro Cell-Migration Assays}

To evaluate the anti-migration effects of drug-loaded liposomes, 2D scratch assay was performed on the monolayer of BEL-7402 and co-cultured systems (Figure 5A). As shown in Figure $5 \mathrm{~B}$ and $\mathrm{C}$, compared to the control group, cell migration inhibition was observed in the cells treated with different drug formulations. As expected, the wound areas in liposomal formulation groups were less than that of free DOX, indicating that DOX\&CA4P-loaded LPs showed a greater anti-migration ability than free DOX. Moreover, 
A

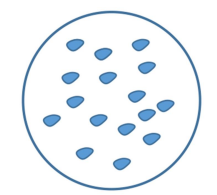

BEL-7402 alone

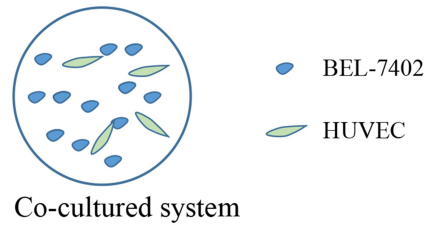

Co-cultured system
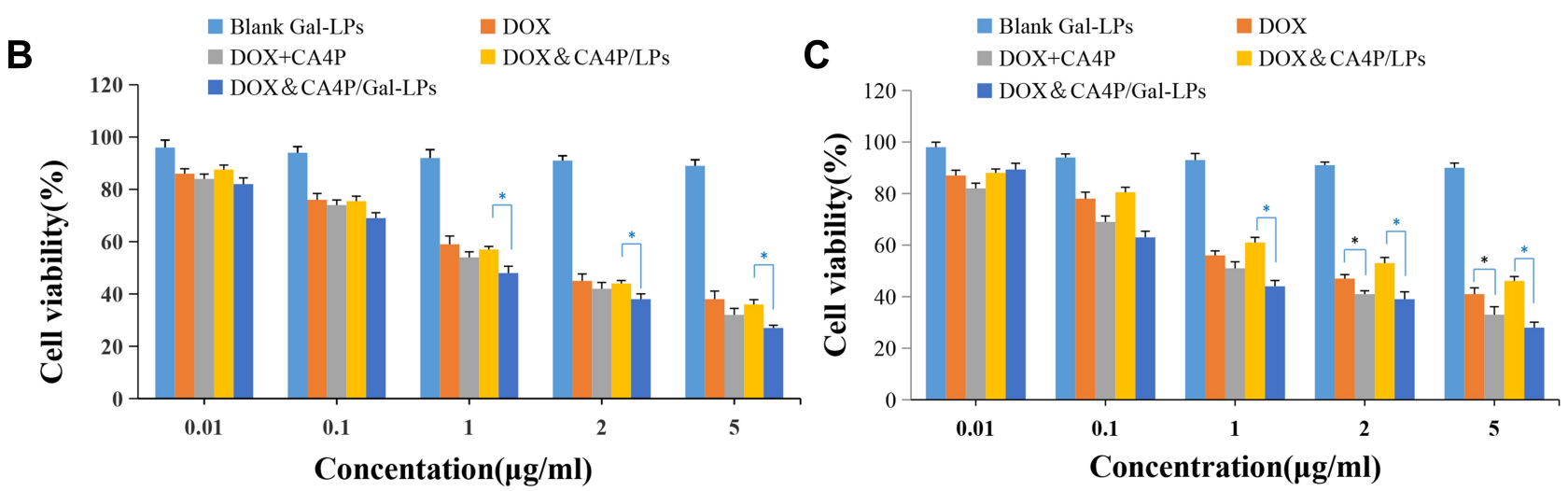

Figure 4 (A) In vitro anti-tumor model of BEL-7402 alone and co-cultured system of BEL-7402 and HUVEC cells. The cytotoxicity of different drug formulations against BEL-7402 alone (B) and co-cultured system (C), respectively. *Value presents statistically significant difference $(P<0.05)$.

the degree of cell migration in DOX\&CA4P/Gal-LPs was lower than that of mixture of DOX and CA4P or DOX\&CA4P/LPs (Figure 5C and D), suggesting that Galmodified LPs promoted the ability of inhibiting cell migration. ${ }^{35}$ Furthermore, as shown in Figure 5C, the green fluorescence-label cells were distributed in the cocultured system, indicating that BEL-7402 and HUVEC cells could grow well together. Interestingly, a mixture of
A

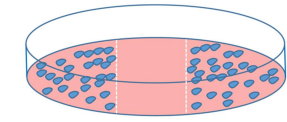

BEL-7402 cells alone

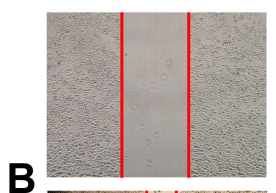

B
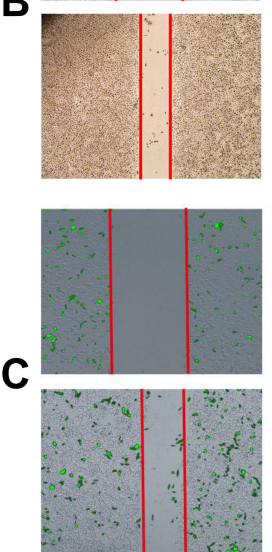

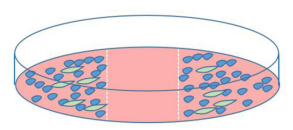

Co-cultured system
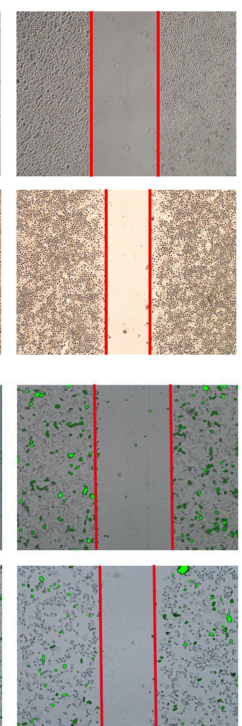
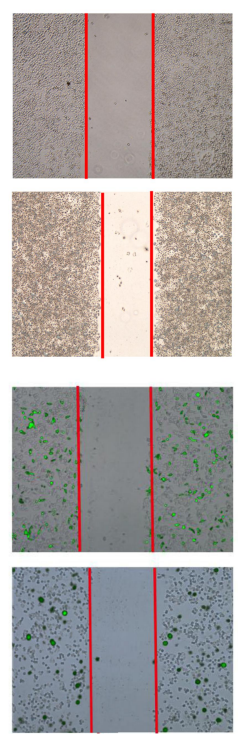

- Bel-7402

$\propto$ HUVEC
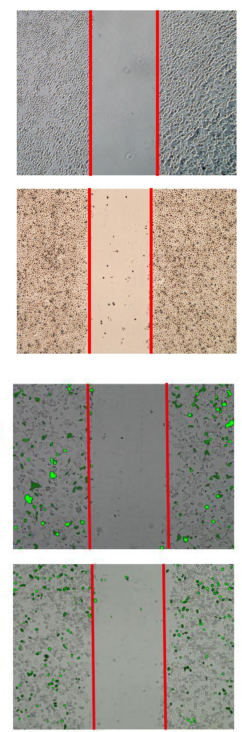
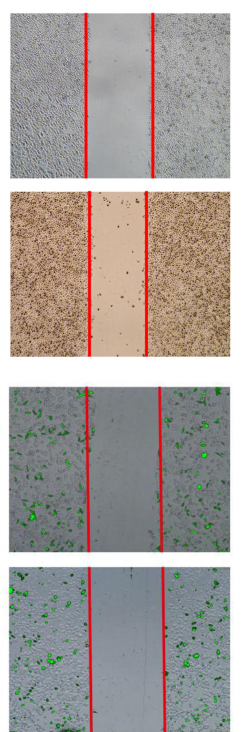
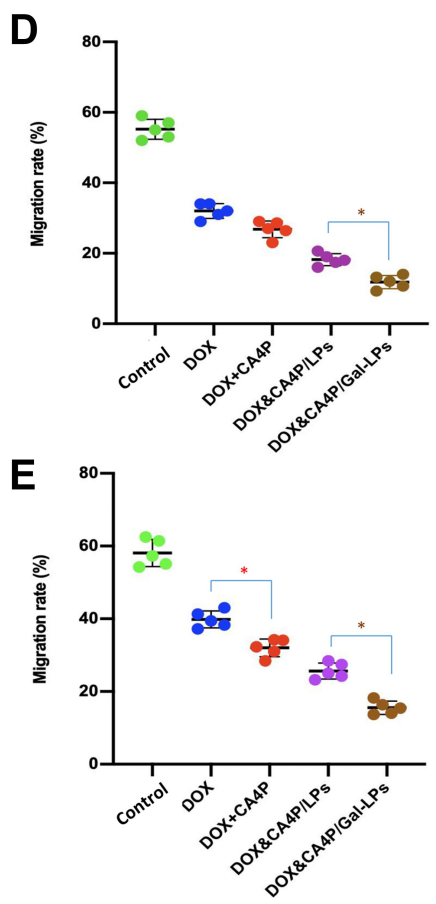

Figure 5 (A) The in vitro cell migration model of BEL-7402 cells alone and co-cultural system of BEL-7402 and HUVEC cells. Typical images of different treatment groups inhibiting cell migration of (B) BEL7402 cells and (C) co-cultured system (green colors represented CFSE-labeled HUVEC cells). The quantification of migration ability were presented as percentages of the wound area in (D) BEL7402 cells alone and (E) co-cultured system. *Value presents statistically significant difference $(P<0.05)$. 
DOX and CA4P showed greater inhibition of cell migration than free DOX in the co-cultured system (Figure 5C and E), while there was no significant difference in the BEL-7402 cells alone, indicating that the co-cultured system was a more effective model to evaluate the anti-tumor activity of combined drugs in vitro. The results showed that combination therapy could exhibit stronger anti-migration effects by pro-apoptotic and anti-angiogenic activities. ${ }^{36}$

\section{In vivo Real-Time Imaging}

To investigate the bio-distribution of the drug in tumorbearing mice, in vivo real-time NIRF imaging of DiR/GalLPs was observed. In this study, DiR, a fluorescence probe, was loaded into liposomes to mimic DOX. The intensity of the fluorescence signals represents the accumulation of the drug in mice. As shown in Figure 6, there was an obvious difference in the fluorescence intensity between free DiR and DiR/Gal-LPs group. At 2 hours, the fluorescence signals in the tumor region could be detected in two groups, and it became stronger in the DiR/Gal-LPs group at 12 hours compared with the free DiR group, suggesting that the GalLPs promoted DiR accumulation in the tumor. At 24 or 48 hours, the fluorescence signals in the Gal-LPs group were still strong, indicating the presence of amounts of DiR in the tumor region. In contrast, there was little fluorescence in the free DiR group. The results showed that Gal-LPs could enhance the drug accumulation in $\mathrm{HCC}$, and keep sustained release at 48 hours. $^{30}$

\section{In vivo Anti-Hepatoma Efficacy}

Tumor proliferation and angiogenesis could promote tumor development, and the combination therapy with DOX and CA4P was used for anti-tumor therapy by proapoptotic and anti-angiogenic activities. The anti-hepatoma efficacy of drug-loaded liposomes was investigated on the H22 cellbearing mice. As shown in Figure 7A, there were obvious decreases of body weight in the groups treated by free DOX or a mixture of DOX and CA4P in comparison with the saline group. By contrast, no significant difference was observed between the liposomal groups and the control group, suggesting that Gal-LPs were biocompatible, and could be used as the safe nano-sized carrier for delivery of anti-tumor drugs. It was shown that the anti-tumor effects of combined treatment groups (mixture of DOX and CA4P, DOX\&CA4P/LPs, and DOX\&CA4P/Gal-LPs) were stronger than that of the single drug group (free DOX), indicating that the addition of CA4P improved the inhibitory effect of DOX in anti-hepatoma therapy (Figure 7B and C). As anticipated, the tumor volumes of the mice treated with two liposomal formulations were smaller than the mixture of DOX and CA4P. The results were due to the fact that the nano-sized carriers could improve the drug accumulation in the tumor region via EPR effect, resulting in enhanced antitumor effects. ${ }^{37}$ Furthermore, DOX\&CA4P/Gal-LP showed superior anti-tumor effects among the four drug treatment groups (Figure 7D), suggesting that introduction
$2 \mathrm{~h}$

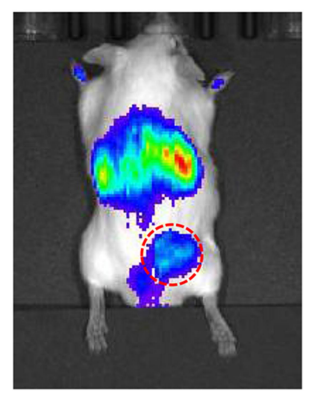

DiR

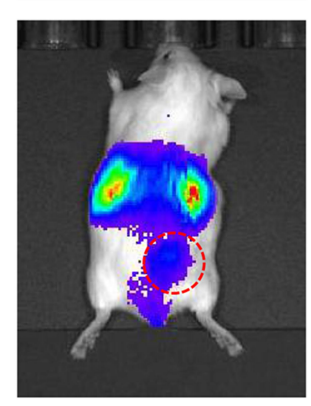

$4 \mathrm{~h}$
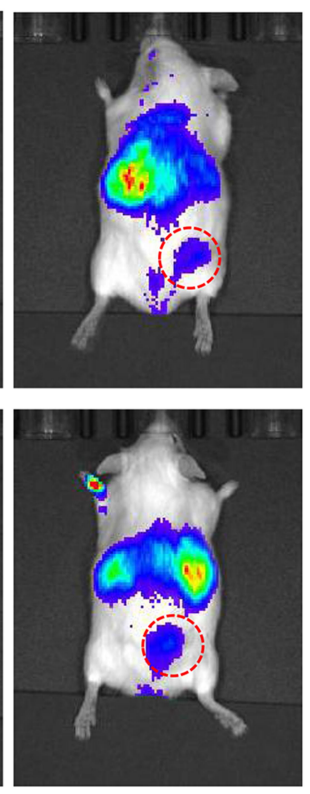

$12 \mathrm{~h}$
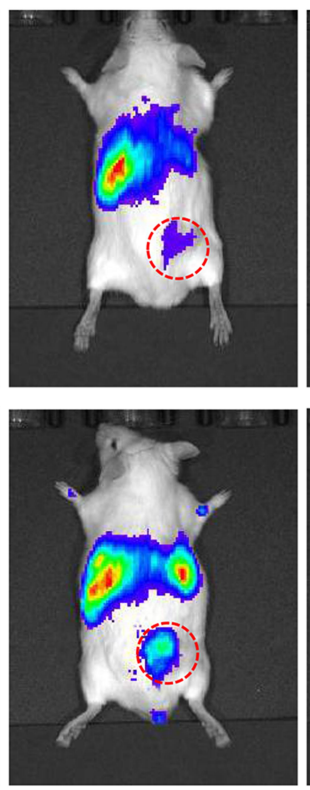

$24 \mathrm{~h}$
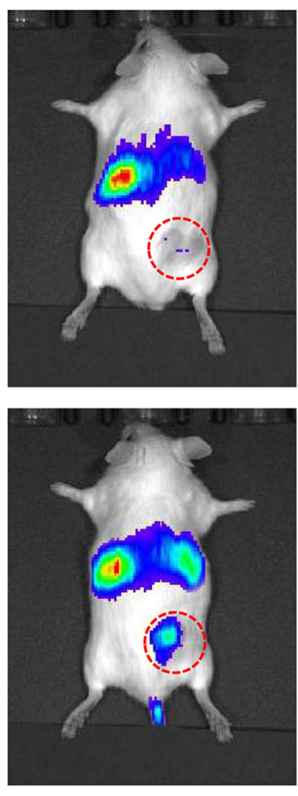

$48 \mathrm{~h}$
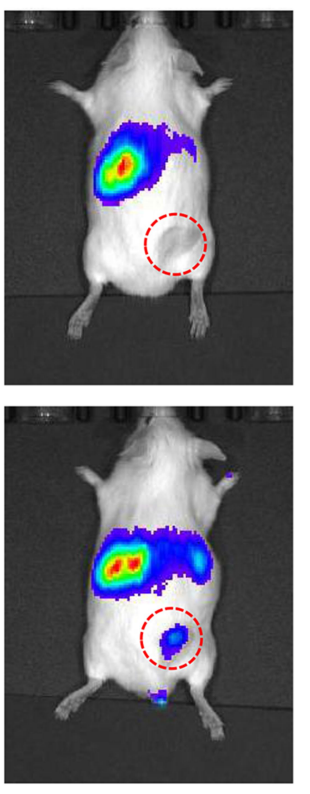

Figure 6 NIRF images of $\mathrm{H} 22$ tumor-bearing mice treated with free DiR or DiR/Gal-LPs. Red circle indicates the tumor region. 
A

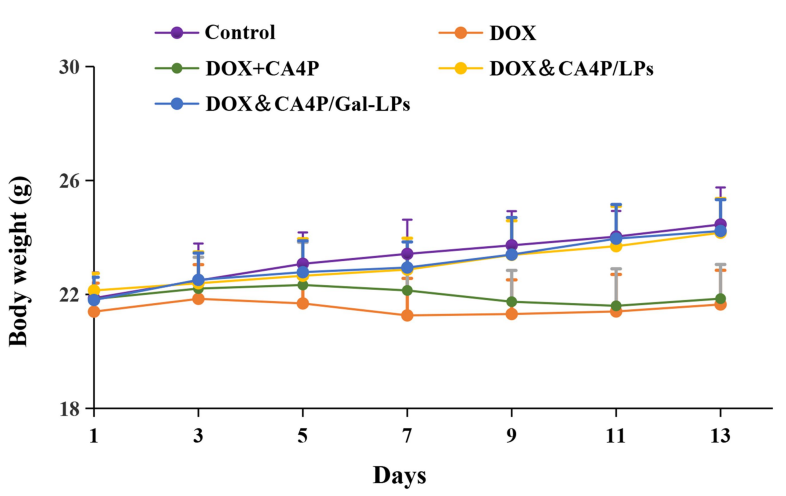

B

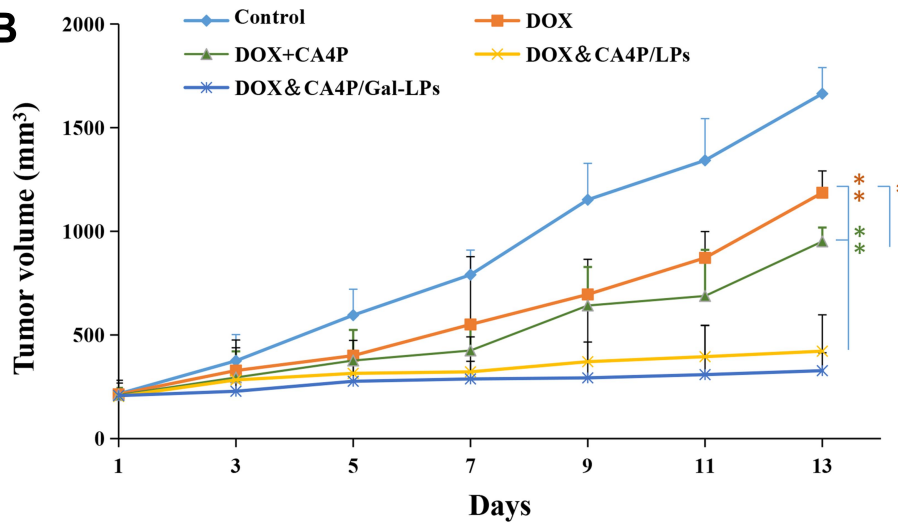

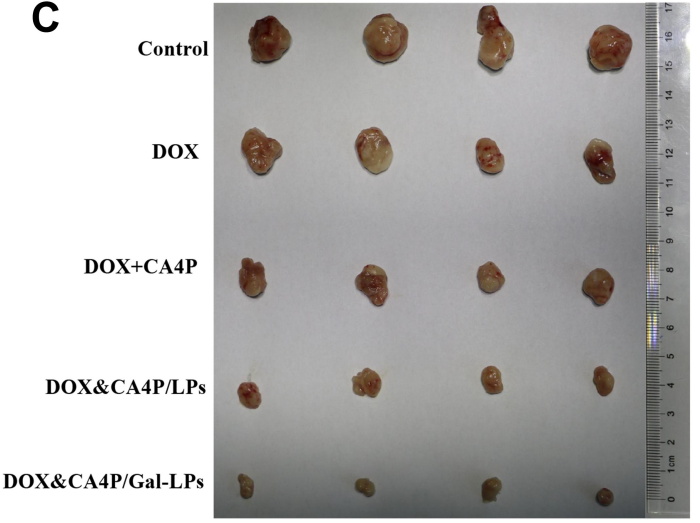

D

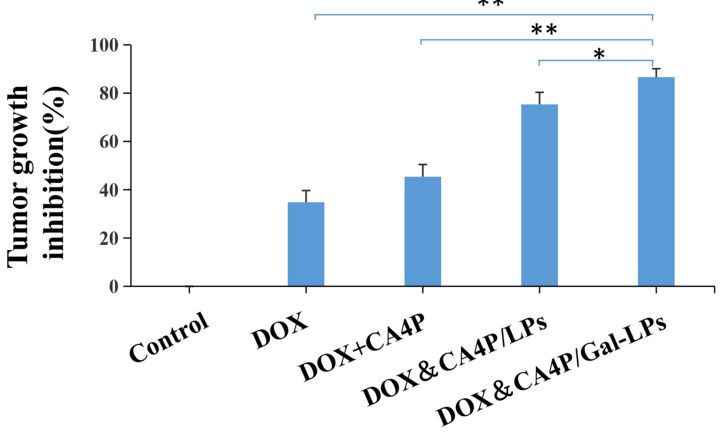

Figure 7 In vivo antitumor effects of different drug formulations. (A) The changes in the body weight of H22 cell-bearing mice; (B) Tumor growth curve during different drug formulations treatment; (C) Tumor images of different groups; (D) Tumor growth inhibition rate $(n=4)$. $* P<0.05, * * P<0.01$.

of Gal molecular promoted the cellular uptake of drugs, leading to superior anti-hepatoma efficacy. ${ }^{38,39}$

\section{Immunohistochemistry and Histological Analysis}

Systemic toxicity of DOX limited its clinical application. Compared with the control group, there was obvious intercellular vacuolation of myocardial fibers in the mice treated by free DOX or a mixture of DOX and CA4P (Figure 8A), suggesting traditional DOX formulation could induce severe cardiotoxicity. By contrast, the mice treated with liposomal formulations (DOX\&CA4P/LPs, DOX\&CA4P/ Gal-LPs) showed no obvious degeneration of myocardial fibers, indicating that the nano-sized liposomes could decrease the systemic toxicity of DOX. ${ }^{40}$

The immunohistochemistry and histological analysis were further performed to evaluate the anti-tumor effects of combination therapy with DOX and CA4P. As shown in Figure 8B, more karyolitic and cytoplasmic vacuolization occurred in tumor areas in the drug-loaded liposomes groups than that in the traditional drug groups (free DOX, mixture of DOX and CA4P), indicating the nano-sized liposomes could improve the anti-tumor efficacy. Additionally, the expression of CD31 (endothelial cell marker) was analyzed by immunohistochemistry. Figure 8C and D showed that there were fewer blood vasculatures in combined drug formulation groups than that in the single DOX group, indicating combination therapy with DOX and CA4P improved anti-angiogenic effects. Compared with DOX\&CA4P/LPs, DOX\&CA4P/Gal-LPs exhibited stronger inhibition of tumor angiogenesis, indicating that GalLPs could promote the anti-angiogenic effect via activetargeting delivery of DOX and CA4P.

\section{Conclusion}

In this study, the novel Gal-modified liposomes (DOX\&CA4P/Gal-LPs) were prepared for anti-hepatoma therapy through the combination of DOX and CA4P. DOX could act on tumor cells to promote the apoptosis of the tumor, while CA4P could perform the anti-angiogenic activities by acting on abnormal blood vessels in the tumor microenvironment. Meanwhile, the Gal molecule could specifically target the ASGP receptor, which is over-expressed on the surface of HCC cells, therefore improving the cellular uptake of liposomes, as well as improving the drug accumulation in tumor 


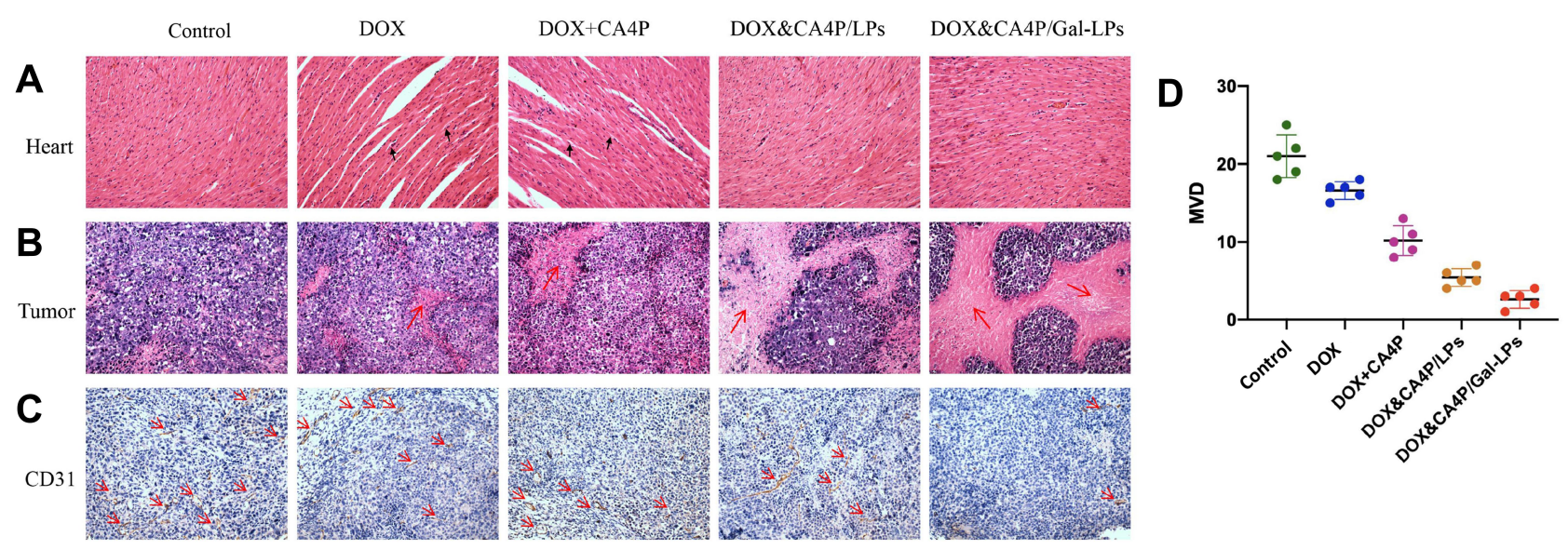

Figure 8 The histological images of Heart (A) and Tumor (B) by H\&E staining; the Immunohistochemistry analysis by MVD images (C) and quantitative data (D). Notes: Black arrows indicate vacuolation of myocardial fibers in (A) and red arrows indicate tumor necrotic area in (B) and microvessels in (C), respectively.

region in vivo. The in vivo anti-tumor results showed that DOX\&CA4P/Gal-LPs exhibited lower tumor angiogenesis, superior anti-proliferation efficacy, and less side-effects. On the other hand, to evaluate the combined anti-tumor effects, a novel co-cultured system composed of BEL-7402 and HUVEC cells was established. Compared with BEL-7402 alone, there were obvious differences in cytotoxicity and antimigration effect between DOX and DOX $+\mathrm{CA} 4 \mathrm{P}$ in the cocultured system, indicating that the co-cultured system was an effective model for evaluation of combination therapy based on pro-apoptotic and anti-angiogenic activities. In summary, Gal-LPs were the potential liver-targeting nanocarriers, and the combination therapy of DOX and CA4P was a promising anti-tumor strategy for HCC treatment.

\section{Acknowledgments}

This study was supported by the National Science Foundation of China (81803464), Natural Science Foundation of Shandong Province (ZR2019BH036), Science and Technology Development Project of Weifang (No. 2018YX058).

\section{Disclosure}

The authors declare that they have no conflicts of interest in this work.

\section{References}

1. Mahvi DA, Liu R, Grinstaff MW, et al. Local cancer recurrence: the realities, challenges, and opportunities for new therapies. $C A$ Cancer J Clin. 2018;68(6):488-505. doi:10.3322/caac.21498

2. Kemp JA, Shim MS, Heo CY, et al. "Combo" nanomedicine: codelivery of multi-modal therapeutics for efficient, targeted, and safe cancer therapy. Adv Drug Deliv Rev. 2016;98:3-18. doi:10.1016/j. addr.2015.10.019
3. Jang B, Kwon H, Katila P, et al. Dual delivery of biological therapeutics for multimodal and synergistic cancer therapies. Adv Drug Deliv Rev. 2016;98:113-133. doi:10.1016/j.addr.2015.10.023

4. Wang Z, Dabrosin C, Yin X, et al. Broad targeting of angiogenesis for cancer prevention and therapy. Semin Cancer Biol. 2015;35 (Suppl):S224-S243. doi:10.1016/j.semcancer.2015.01.001

5. Van der Veldt AA, Lubberink M, Bahce I, et al. Rapid decrease in delivery of chemotherapy to tumors after anti-VEGF therapy: implications for scheduling of anti-angiogenic drugs. Cancer Cell. 2012;21 (1):82-91. doi:10.1016/j.ccr.2011.11.023

6. Li F, Wang Y, Chen WL, et al. Co-delivery of VEGF siRNA and etoposide for enhanced anti-angiogenesis and anti-proliferation effect via multi-functional nanoparticles for orthotopic non-small cell lung cancer treatment. Theranostics. 2019;9(20):5886-5898. doi:10.7150/thno.32416

7. von Minckwitz G, Eidtmann H, Rezai M, et al. Neoadjuvant chemotherapy and bevacizumab for HER2-negative breast cancer. N Engl J Med. 2012;366(4):299-309. doi:10.1056/NEJMoa1111065

8. Tewari KS, Sill MW, Long HJ, et al. Improved survival with bevacizumab in advanced cervical cancer. $N$ Engl J Med. 2014;370 (8):734-743. doi:10.1056/NEJMoa1309748

9. Carmeliet P, Jain RK. Molecular mechanisms and clinical applications of angiogenesis. Nature. 2011;473(7347):298-307. doi:10.1038/ nature 10144

10. Abdollahi A, Folkman J. Evading tumor evasion: current concepts and perspectives of anti-angiogenic cancer therapy. Drug Resist Updat. 2010;13(1-2):16-28. doi:10.1016/j.drup.2009.12.001

11. Li Z, Di C, Li S, et al. Smart nanotherapeutic targeting of tumor vasculature. Acc Chem Res. 2019;52(9):2703-2712. doi:10.1021/acs. accounts. $9 \mathrm{~b} 00283$

12. Yang S, Tang Z, Hu C, et al. Selectively potentiating hypoxia levels by combretastatin A4 nanomedicine: toward highly enhanced hypoxia-activated prodrug tirapazamine therapy for metastatic tumors. Adv Mater. 2019;31(11):e1805955. doi:10.1002/adma.201805955

13. Dai W, Wang X, Song G, et al. Combination antitumor therapy with targeted dual-nanomedicines. Adv Drug Deliv Rev. 2017;115:23-45. doi:10.1016/j.addr.2017.03.001

14. Liu T, Zhang D, Song W, et al. A poly(1-glutamic acid)-combretastatin A4 conjugate for solid tumor therapy: markedly improved therapeutic efficiency through its low tissue penetration in solid tumor. Acta Biomater. 2017;53:179-189. doi:10.1016/j.actbio.2017.02.001

15. Shen N, Wu J, Yang C, et al. Combretastatin A4 nanoparticles combined with hypoxia-sensitive imiquimod: a new paradigm for the modulation of host immunological responses during cancer treatment. Nano Lett. 2019;19(11):8021-8031. doi:10.1021/acs. nanolett.9b03214 
16. Byrne JD, Jajja MR, O’Neill AT, et al. Local iontophoretic administration of cytotoxic therapies to solid tumors. Sci Transl Med. 2015;7 (273):273ra214. doi:10.1126/scitranslmed.3009951

17. Orecchioni M, Cabizza R, Bianco A, et al. Graphene as cancer theranostic tool: progress and future challenges. Theranostics. 2015;5(7):710-723. doi:10.7150/thno.11387

18. Shim G, Kim MG, Kim D, et al. Nanoformulation-based sequential combination cancer therapy. Adv Drug Deliv Rev. 2017;115:57-81. doi:10.1016/j.addr.2017.04.003

19. Dutta R, Mahato RI. Recent advances in hepatocellular carcinoma therapy. Pharmacol Ther. 2017;173:106-117. doi:10.1016/j. pharmthera.2017.02.010

20. $\mathrm{Mu} \mathrm{W}$, Jiang $\mathrm{D}, \mathrm{Mu} \mathrm{S}$, et al. Promoting early diagnosis and precise therapy of hepatocellular carcinoma by glypican-3-targeted synergistic chemo-photothermal theranostics. ACS Appl Mater Interfaces. 2019;11(26):23591-23604. doi:10.1021/acsami.9b05526

21. Wang J, Wu Z, Pan G, et al. Enhanced doxorubicin delivery to hepatocellular carcinoma cells via CD147 antibody-conjugated immunoliposomes. Nanomed Nanotechnol Biol Med. 2018;14 (6):1949-1961. doi:10.1016/j.nano.2017.09.012

22. Trere D, Fiume L, De Giorgi LB, et al. The asialoglycoprotein receptor in human hepatocellular carcinomas: its expression on proliferating cells. $\mathrm{Br} J$ Cancer. 1999;81(3):404-408. doi:10.1038/sj. bjc. 6690708

23. Zhang T, Li G, Li S, et al. Asialoglycoprotein receptor targeted micelles containing carborane clusters for effective boron neutron capture therapy of hepatocellular carcinoma. Colloids Surf $B$ Biointerfaces. 2019;182:110397. doi:10.1016/j.colsurfb.201 9.110397

24. Jiang H, Li ZP, Tian GX, et al. Liver-targeted liposomes for codelivery of curcumin and combretastatin A4 phosphate: preparation, characterization, and antitumor effects. Int $J$ Nanomed. 2019;14:1789-1804. doi:10.2147/IJN.S188971

25. Zhang B, Wang T, Yang S, et al. Development and evaluation of oxaliplatin and irinotecan co-loaded liposomes for enhanced colorectal cancer therapy. $J$ Control Release. 2016;238:10-21. doi:10.1016/j.jconrel.2016.07.022

26. Madamsetty VS, Sharma A, Toma M, et al. Tumor selective uptake of drug-nanodiamond complexes improves therapeutic outcome in pancreatic cancer. Nanomed-Nanotechnol. 2019;18:112-121. doi:10.1016/j.nano.2019.02.020

27. McMahon EJ, Bailey SL, Castenada CV, et al. Epitope spreading initiates in the CNS in two mouse models of multiple sclerosis. Nat Med. 2005;11(3):335-339. doi:10.1038/nm1202

28. Alipour E, Halverson D, McWhirter S, et al. Phospholipid bilayers: stability and encapsulation of nanoparticles. Annu Rev Phys Chem. 2017;68:261-283. doi:10.1146/annurev-physchem-040215-112634
29. Chountoulesi M, Demetzos C. Promising nanotechnology approaches in treatment of autoimmune diseases of central nervous system. Brain Sci. 2020;10(6):338. doi:10.3390/brainsci10060338

30. Cao J, Gao X, Cheng M, et al. Reversible shielding between dual ligands for enhanced tumor accumulation of ZnPc-Loaded micelles. Nano Lett. 2019;19(3):1665-1674. doi:10.1021/acs.nanolett.8b04645

31. Li Y, Miao Y, Chen M, et al. Stepwise targeting and responsive lipid-coated nanoparticles for enhanced tumor cell sensitivity and hepatocellular carcinoma therapy. Theranostics. 2020;10 (8):3722-3736. doi:10.7150/thno.42008

32. Zhou Q, Zhou Y, Liu X, Shen Y. GDC-0449 improves the antitumor activity of nano-doxorubicin in pancreatic cancer in a fibroblast-enriched microenvironment. Sci Rep. 2017;7(1):13379. doi:10.1038/s41598-017-13869-0

33. Malam Y, Loizidou M, Seifalian AM. Liposomes and nanoparticles: nanosized vehicles for drug delivery in cancer. Trends Pharmacol Sci. 2009;30(11):592-599. doi:10.1016/j.tips.2009.08.004

34. Jiang S, Li M, Hu Y, et al. Multifunctional self-assembled micelles of galactosamine-hyaluronic acid-vitamin E succinate for targeting delivery of norcantharidin to hepatic carcinoma. Carbohydr Polym. 2018;197:194-203. doi:10.1016/j.carbpol.2018.05.090

35. Huang Y, Hu L, Huang S, et al. Curcumin-loaded galactosylated BSA nanoparticles as targeted drug delivery carriers inhibit hepatocellular carcinoma cell proliferation and migration. Inter $J$ Nanomed. 2018;13:8309-8323. doi:10.2147/IJN.S184379

36. Heindryckx F, Coulon S, Terrie E, et al. The placental growth factor as a target against hepatocellular carcinoma in a diethylnitrosamine-induced mouse model. J Hepatol. 2013;58 (2):319-328. doi:10.1016/j.jhep.2012.09.032

37. Ojha T, Pathak V, Shi Y, et al. Pharmacological and physical vessel modulation strategies to improve EPR-mediated drug targeting to tumors. Adv Drug Delive Rev. 2017;119:44-60. doi:10.1016/j. addr.2017.07.007

38. Zou Y, Song Y, Yang W, et al. Galactose-installed photo-crosslinked $\mathrm{pH}$-sensitive degradable micelles for active targeting chemotherapy of hepatocellular carcinoma in mice. $J$ Control Release. 2014;193:154-161. doi:10.1016/j.jconrel.2014.05.016

39. $\mathrm{Hu} \mathrm{H}, \mathrm{Xiao} \mathrm{C}, \mathrm{Wu} \mathrm{H}$, et al. Nanocolloidosomes with selective drug release for active tumor-targeted imaging-guided photothermal/ chemo combination therapy. ACS Appl Mater Inter. 2017;9 (48):42225-42238. doi:10.1021/acsami.7b14796

40. Bavli Y, Winkler I, Chen BM, et al. Doxebo (doxorubicin-free Doxil-like liposomes) is safe to use as a pre-treatment to prevent infusion reactions to PEGylated nanodrugs. J Control Release. 2019;306:138-148. doi:10.1016/j.jconrel.2019.06.007
International Journal of Nanomedicine

\section{Publish your work in this journal}

The International Journal of Nanomedicine is an international, peerreviewed journal focusing on the application of nanotechnology in diagnostics, therapeutics, and drug delivery systems throughout the biomedical field. This journal is indexed on PubMed Central, MedLine, CAS, SciSearch ${ }^{\mathbb{R}}$, Current Contents ${ }^{\mathbb{B}} /$ Clinical Medicine,
Journal Citation Reports/Science Edition, EMBase, Scopus and the Elsevier Bibliographic databases. The manuscript management system is completely online and includes a very quick and fair peer-review system, which is all easy to use. Visit http://www.dovepress.com/ testimonials.php to read real quotes from published authors. 